\title{
Estudo bibliométrico da produção científica internacional sobre o uso de produtos naturais como terapia para o câncer de pâncreas
}

Bibliometric study of international scientific production on the use of natural products as a therapy for pancreatic cancer

Estudio bibliométrico de la producción científica internacional sobre el uso de productos naturales como terapia para el cáncer de páncreas

Bruno Mendes Moura Dias Guerra

ORCID: https://orcid.org/0000-0001-9394-5529 Instituto de Educação Superior do Vale do Parnaíba, Brasil E-mail: brunommdguerra@gmail.com

Caliza Calmon de Araújo Mascarenhas ORCID: https://orcid.org/0000-0002-8294-0201 Instituto de Educação Superior do Vale do Parnaíba, Brasil E-mail: calizacalmon_@hotmail.com

Ricardo Vieira Bezerra Luz Rodrigues ORCID: https://orcid.org/0000-0003-1429-9966 Instituto de Educação Superior do Vale do Parnaíba, Brasil

E-mail: ricardobvlrodrigues@hotmail.com

Mateus Nunes Carvalho

ORCID: https://orcid.org/0000-0002-6099-0910 Instituto de Educação Superior do Vale do Parnaíba, Brasil E-mail: mateusnunesc@gmail.com

Raissa Pinheiro Nery

ORCID: https://orcid.org/0000-0003-3578-3896 Instituto de Educação Superior do Vale do Parnaíba, Brasil E-mail: raissa-nery@hotmail.com

Iane Lopes da Rocha

ORCID: https://orcid.org/0000-0002-7589-6775 Instituto de Educação Superior do Vale do Parnaíba, Brasil E-mail: iane_lrocha@hotmail.com

Mario Abel Lima Barros

ORCID: https://orcid.org/0000-0003-0717-2983

Centro Universitário Maurício de Nassau, Brasil

E-mail: marioabel.limabarros@gmail.com

André Luca Araujo de Sousa

ORCID: https://orcid.org/0000-0003-4876-9188 Universidade Estadual do Piauí, Brasil E-mail: a.lucaaraujo10@gmail.com

Antonione Santos Bezerra Pinto

ORCID: https://orcid.org/0000-0002-6577-2816 Instituto de Educação Superior do Vale do Parnaíba, Brasil E-mail: antonione182@hotmail.com

\section{Resumo}

Introdução: Devido ao alto índice de invasão, malignidade e desenvolvimento assintomático, o câncer de pâncreas é altamente letal. Uma pequena quantidade de drogas está disponível para sua abordagem. Objetivo: Diante disso, o presente estudo objetiva mapear a produção científica internacional sobre o uso de produtos naturais como terapia para o câncer de pâncreas. Método: Foi realizada uma pesquisa bibliométrica em trabalhos acadêmicos no Web of Science publicados de 1998 a 2019 usando os termos de busca: "pancreatic cancer", "therapy" e "natural product". Foram identificados, após aplicados os filtros de refinamento, 123 registros de publicação em 82 periódicos distintos indexados à base de dados em questão, escritos por 755 autores que possuem vínculos à 237 instituições, localizadas em 37 países. Resultado: Avaliou-se a distribuição da quantidade de publicações por ano, os periódicos mais citados e mais relacionados entre si, os top periódicos com mais citações, a quantidade de artigos por país de origem das instituições de vínculo dos autores, os artigos mais citados e a co-ocorrência de palavras mais citadas e mais relacionados entre si. Conclusão: Embora publicações recentes tenham identificado mecanismos de ação envolvidos 
na interação de variados produtos naturais com o câncer de pâncreas, estamos longe de entender o espectro de efeitos que a terapêutica natural exerce sobre as células normais e cancerígenas. Novos estudos sobre o tema deverão ser realizados e a quantidade e qualidade das publicações acerca do tema deverão crescer ainda mais.

Palavras-chave: Bibliometria; Neoplasias pancreáticas; Produtos biológicos; Tratamento farmacológico.

\begin{abstract}
Introduction: Due to the high rate of invasion, malignancy and asymptomatic development, pancreatic cancer is highly lethal. A small number of drugs is available for its approach. Objective: Given this, the present study aims to map the international scientific production on the use of natural products as therapy for pancreatic cancer. Method: Bibliometric research was carried out on academic works on the Web of Science published from 1998 to 2019 using the search terms: "pancreatic cancer", "therapy" and "natural product". After applying the refinement filters, 123 publication records were identified in 82 different journals indexed to the database in question, written by 755 authors who have links to 237 institutions, located in 37 countries. Result: The distribution of the number of publications per year, the most cited and most related journals, the top journals with the most citations, the number of articles by country of origin of the authors' link institutions, the most cited articles were evaluated. and the co-occurrence of the most cited and most related words. Conclusion: Although recent publications have identified mechanisms of action involved in the interaction of various natural products with pancreatic cancer, we are far from understanding the spectrum of effects that natural therapy has on normal and cancer cells. Further studies on the topic are expected to be carried out and the quantity and quality of publications on the topic are expected to grow even more.
\end{abstract}

Keywords: Bibliometrics; Pancreatic neoplasms; Biological products; Drug therapy.

\title{
Resumen
}

Introducción: Debido a la alta tasa de invasión, malignidad y desarrollo asintomático, el cáncer de páncreas es altamente letal. Hay una pequeña cantidad de medicamentos disponibles para su enfoque. Objetivo: Por lo tanto, este estudio tiene como objetivo mapear la producción científica internacional sobre el uso de productos naturales como terapia para el cáncer de páncreas. Método: Se realizó una búsqueda bibliométrica de artículos académicos en la Web of Science publicados entre 1998 y 2019 utilizando los términos de búsqueda: "cáncer de páncreas", "terapia" y "producto natural". Después de aplicar los filtros de refinamiento, se identificaron 123 registros de publicación en 82 revistas diferentes indexadas a la base de datos en cuestión, redactadas por 755 autores que tienen enlaces a 237 instituciones ubicadas en 37 países. Resultados: Se evaluó la distribución del número de publicaciones por año, las revistas más citadas y más relacionadas, las principales revistas con más citas, el número de artículos por país de origen de las instituciones de afiliación de los autores, los artículos más citados y la co-ocurrencia de las palabras más citadas y más relacionadas. Conclusión: Aunque publicaciones recientes han identificado mecanismos de acción involucrados en la interacción de varios productos naturales con el cáncer de páncreas, estamos lejos de comprender el espectro de efectos que tiene la terapia natural sobre las células normales y cancerosas. Deben realizarse nuevos estudios sobre el tema y aumentar aún más la cantidad y calidad de las publicaciones sobre el tema.

Palabras clave: Bibliometría; Neoplasias pancreáticas; Productos biológicos; Quimioterapia.

\section{Introdução}

O câncer de pâncreas é um tipo de tumor maligno comum, prevalente em todo o mundo com alto índice de invasão, malignidade e desenvolvimento assintomático sendo altamente letal (Siegel, Miller \& Jemal, 2020). Segundo dados do Sistema de Informação sobre Mortalidade do Ministério da Saúde e do Atlas On-line de Mortalidade do Instituto nacional do Câncer (INCA), 95.976 pessoas vieram a óbito por câncer de pâncreas no Brasil (ATLAS, 2020).

Dados da Global Cancer Statistics em 2020 indicaram que o câncer de pâncreas foi a sétima principal causa de morte por câncer em homens e mulheres em todo o mundo e teve a pior taxa de sobrevida em 5 anos dentre todos os cânceres, sobrevivendo apenas 9 a cada 100 pacientes neste período (Siegel, Miller \& Jemal, 2020).

Pesquisadores da china analisaram tendências temporais de 1973 a 2015 em 41 países, incluindo o Brasil e observaram aumento na incidência de câncer de pâncreas em homens e mulheres na maioria dos países pesquisados. Embora haja um declínio da prevalência do tabagismo, os autores apontaram que o aumento da prevalência da obesidade e do diabetes, o consumo crescente de carne vermelha e processada, a diminuição da ingesta de frutas e vegetais e o aumento do sedentarismo são fatores contribuintes para este aumento na incidência (Luo et al., 2019). 
Apesar das recentes melhorias nas técnicas de diagnóstico, a maioria dos pacientes com câncer de pâncreas é diagnosticada em estados avançados. Grande parte dos pacientes morrem dentro de um ano após o diagnóstico. A cirurgia é uma abordagem terapêtica curativa essencial para a maioria dos pacientes com câncer de pâncreas. No entanto, a taxa de sucesso da cirurgia de ressecção permanece muito baixa, visto que cerca de $80 \%$ a $85 \%$ dos pacientes com este diagnóstico já estão em estágio avançado. Visto isso, apenas $15 \%$ a $20 \%$ dos pacientes com câncer de pâncreas são elegíveis para ressecção cirúrgica após serem diagnosticados. Os procedimentos cirúrgicos padrão incluem pancreatoduodenectomia (preservação do piloro ou preservação do estômago) para tumores da cabeça do pâncreas e pancreatectomia distal com esplenectomia para tumores que surgem na cauda ou no corpo do pâncreas (Paulson et al., 2013). A ressecção radical, isoladamente ou em combinação com outra terapia, é a única maneira de erradicar o câncer de pâncreas. Apenas 10\% a 20\% dos pacientes, passam por ressecção radical podem sobreviver 5 anos. Portanto, é necessário destacar a necessidade de melhor estadiamento préoperatório e terapias sistêmicas mais eficazes (Perkhofer, Ettrich \& Seufferlein, 2015).

A quimioterapia e radioterapia são consideradas as abordagens padrão de tratamento para pacientes com câncer de pâncreas irressecável, especialmente para pacientes em estágios avançados ou metastáticos inoperáveis. Nas últimas três décadas, os medicamentos terapêuticos padrão para o câncer de pâncreas foram o fluoropirimidina, o 5 - fluorouracil (5-FU) e o antimetabolito gemcitabina (Perkhofer, Ettrich \& Seufferlein, 2015).

Devido à alta mortalidade e à pequena quantidade de drogas disponíveis para a abordagem e tratamento do câncer de pâncreas, produtos fitoterápicos vêm sendo estudados como novas terapias adjuvantes e neoadjuvantes para o tratamento deste.

Diante disso, o presente estudo tem como objetivo mapear a produção científica internacional sobre o uso de produtos naturais como terapia para o câncer de pâncreas.

\section{Metodologia}

O estudo trata-se de um estudo qualitativo e quantitativo a partir de um levantamento bibliográfico, além de uma de revisão integrativa a respeito do tema para satisfazer o objetivo do estudo (Souza, Silva \& Carvalho, 2010)

Foram usados os termos de busca: ("pancreatic cancer*" and "therapy*" and "natural product*") (o asterisco possibilita a busca nos plurais das palavras, além de outros sufixos) na base de dados ISI Web of Knowledge/Web of Science (WoS), no recorte temporal entre os anos 1998 e 2019, analisando os registros que apresentam os termos selecionados para a busca, com base em revisão de literatura sobre a temática em questão. Como percurso metodológico, foi realizada uma pesquisa bibliométrica em trabalhos acadêmicos no WoS, identificando-se após aplicação dos filtros de refinamento, 123 registros de publicação em 82 periódicos distintos.

Os critérios de inclusão para este estudo bibliométrico foram os seguintes: a) artigos de periódicos publicados que abordavam o uso de produtos naturais como novas terapias para o câncer de pâncreas, b) idioma da publicação em inglês; c) foram incluídos artigos, independentemente de sua metodologia; d) estudos publicados entre $1^{\circ}$ de janeiro de 1998, até 31 de dezembro de 2019 foram incluídos. Além disso, foram excluídos se tivessem conflitos a algum dos itens dos critérios de inclusão.

\section{Resultados e Discussão}

Após realizado o levantamento bibliométrico na principal coleção do WoS, foram identificados 123 artigos sobre o uso de produtos naturais como terpia para o câncer de pâncreas. Estes artigos estão publicados em 82 periódicos distintos indexados à base de dados em questão e foram escritos por 755 autores vinculados à 237 instituições localizadas em 37 países. 
Para a consecução destes artigos foram utilizadas 9.884 referências, com uma média de aproximadamente 80,4 referências por artigo (Tabela 1).

Tabela 1: Resultados Gerais do Levantamento Bibliométrico (1998-2019).

\begin{tabular}{lc}
\hline Dados Bibliométricos & Quantidade \\
\hline Publicações (artigos) & 123 \\
Periódicos indexados & 82 \\
Autores & 748 \\
Instituições (vínculos dos autores) & 237 \\
Países & 37 \\
Referências citadas & 9884 \\
\hline
\end{tabular}

Fonte: Elaboração própria com apoio do software HistCite a partir de dados da Web of Science $e^{T M}$.

Dos dados levantados, verificou-se um aumento crescente na quantidade de artigos publicados sobre o tema ao longo dos anos, como pode ser visto na Grafico 1.

Gráfico 1: Distribuição das publicações (1998-2019).

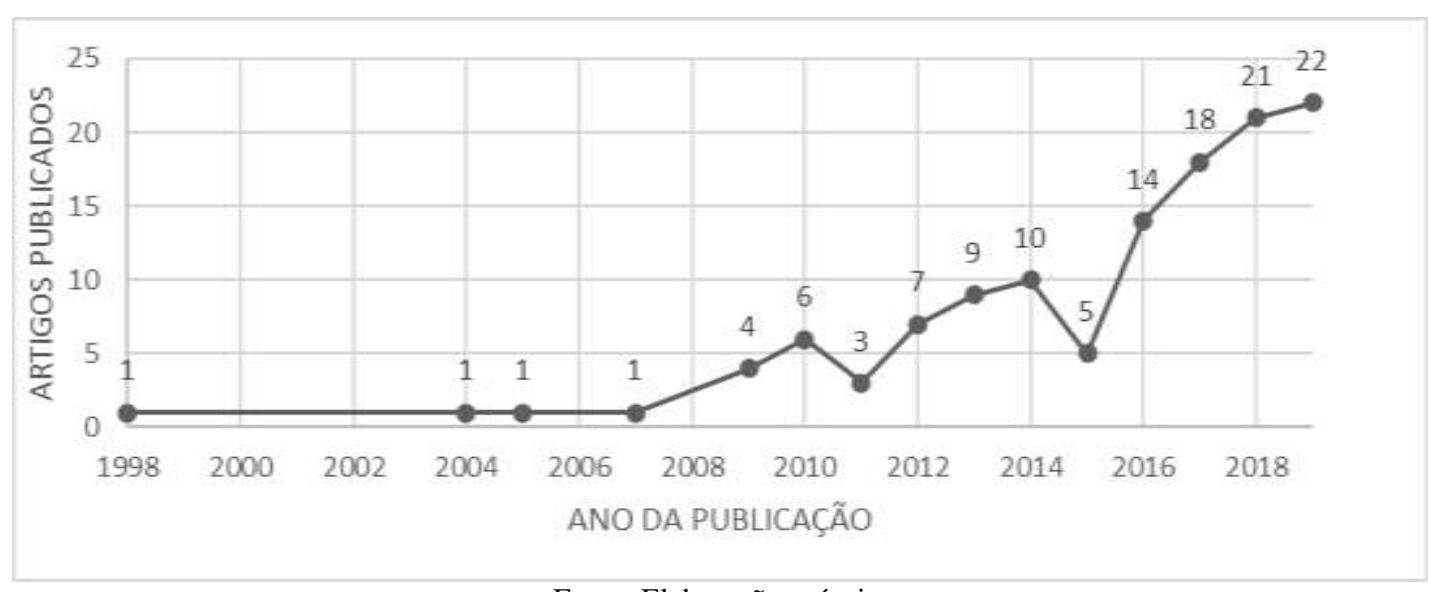

Fonte: Elaboração própria.

Para analisar os periódicos mais citados e mais relacionados entre si, a Figura 1 foi construída. 
Figura 1: Periódicos mais citados e mais relacionados entre si (1998-2019).

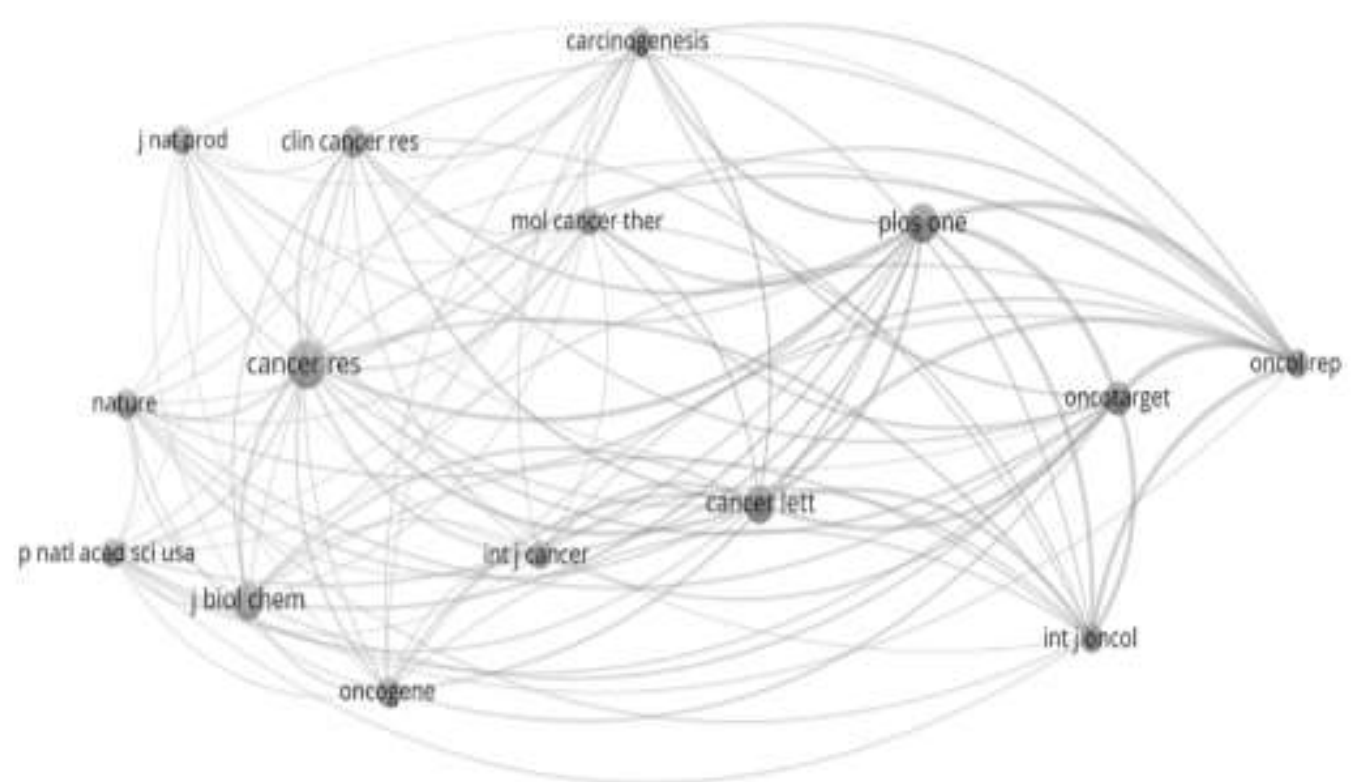

Fonte: Elaboração no software VOSviewer a partir de dados da Web of Science $e^{T M}$

Dos periódicos analisados, 15 haviam recebido 100 ou mais citações dos artigos relacionados ao uso de produtos naturais como terapia para o câncer de pâncreas conforme exposto na Tabela 2.

Tabela 2: Top Periódicos com mais citações (1998-2019).

\begin{tabular}{lcc}
\hline \multicolumn{1}{c}{ Periódicos } & Citaçóes & $\begin{array}{c}\text { Força das } \\
\text { Conexões }\end{array}$ \\
\hline Plos One & 204 & 10226 \\
Oncotarget & 158 & 8954 \\
Oncology Report & 112 & 8303 \\
Cancer Research & 323 & 7829 \\
International Journal of Oncology & 105 & 6961 \\
Cancer Letters & 182 & 6808 \\
The Journal of Biological Chemistry & 184 & 4582 \\
Carcinogenesis & 105 & 4433 \\
Clinical Cancer Research & 142 & 4108 \\
Oncogene & 124 & 3954 \\
International Journal of Cancer & 109 & 3889 \\
Molecular Cancer Therapeutics & 105 & 3592 \\
Nature & 117 & 2980 \\
Proceedings of the National Academy of Sciences & 106 & 2737 \\
Journal of Natural Products & 103 & 1580 \\
\hline
\end{tabular}

Fonte: Elaboração própria com apoio do software VOSviewer a partir de dados da Web of Science $e^{T M}$.

Os estudos selecionados foram escritos por pesquisadores de diversos países sendo que 53 deles são dos Estados Unidos, 34 da China, 12 da Índia, 8 da Coréia do Sul, 6 da Alemanha, 4 do Brasil, 4 do Egito, 3 australianos, 3 iranianos e 3 italianos mostrando que o interesse pela pesquisa do tema é global. 
Os dez artigos mais citados variam entre estudos pré-clínicos, estudos biotecnológicos, vias de ação dos produtos naturais avaliados e potenciais alvos terapêuticos, o que torna esta lista bem diversificada conforme exposto na Tabela 3.

Tabela 3: Artigos mais citados na Web of Science ${ }^{\mathrm{TM}}$

\begin{tabular}{|c|c|c|c|}
\hline Citações & \begin{tabular}{|l} 
Títulos dos artigos \\
\end{tabular} & Fonte das publicações & Autores/Ano \\
\hline 257 & $\begin{array}{l}\text { Curcumin-loaded biodegradable polymeric micelles } \\
\text { for colon cancer therapy in vitro and in vivo }\end{array}$ & Nanoscale & Gou et al. (2010) \\
\hline 192 & $\begin{array}{c}\text { Discovery of a Potent and Orally Active Hedgehog } \\
\text { Pathway Antagonist (IPI-926) }\end{array}$ & $\begin{array}{l}\text { Journal of Medicinal } \\
\text { Chemistry }\end{array}$ & Tremblay et al. (2009) \\
\hline 140 & $\begin{array}{c}\text { Resveratrol inhibits hypoxia-induced accumulation } \\
\text { of hypoxia-inducible factor-1 alpha and VEGF } \\
\text { expression in human tongue squamous cell } \\
\text { carcinoma and hepatoma cells }\end{array}$ & $\begin{array}{l}\text { Molecular Cancer } \\
\text { Therapeutics }\end{array}$ & Zhang et al. (2005) \\
\hline 126 & $\begin{array}{c}\text { Induction of proteasome expression in skeletal } \\
\text { muscle is attenuated by inhibitors of NF-kappa B } \\
\text { activation }\end{array}$ & British Journal of Cancer & Wyke et al. (2004) \\
\hline 83 & $\begin{array}{l}\text { From a Natural Product Lead to the Identification of } \\
\text { Potent and Selective Benzofuran-3-yl-(indol-3- } \\
\text { yl)maleimides as Glycogen Synthase Kinase } 3 \text { beta } \\
\text { Inhibitors That Suppress Proliferation and Survival } \\
\text { of Pancreatic Cancer Cells }\end{array}$ & $\begin{array}{l}\text { Journal of Medicinal } \\
\text { Chemistry }\end{array}$ & Gaisina et al. (2009) \\
\hline 81 & Modulators of the hedgehog signaling pathway & $\begin{array}{l}\text { Bioorganic \& Medicinal } \\
\text { Chemistry }\end{array}$ & Heretsch et al. (2010) \\
\hline 79 & The Role of Resveratrol in Cancer Therapy & $\begin{array}{l}\text { International Journal of } \\
\text { Molecular Sciences }\end{array}$ & Ko et al. (2017) \\
\hline 71 & $\begin{array}{l}\text { Novel ginsenosides } 25-\mathrm{OH}-\mathrm{PPD} \text { and } 25-\mathrm{OCH} 3-\mathrm{PPD} \\
\text { as experimental therapy for pancreatic cancer: } \\
\text { anticancer activity and mechanisms of action }\end{array}$ & Cancer Letters & Wang et al. (2009) \\
\hline 64 & $\begin{array}{l}\text { Graviola: A novel promising natural-derived drug } \\
\text { that inhibits tumorigenicity and metastasis of } \\
\text { pancreatic cancer cells in vitro and in vivo through } \\
\text { altering cell metabolism }\end{array}$ & Cancer Letters & Torres et al. (2012) \\
\hline 55 & $\begin{array}{c}\text { Effects of Thymoquinone in the Expression of Mucin } \\
4 \text { in Pancreatic Cancer Cells: Implications for the } \\
\text { Development of Novel Cancer Therapies }\end{array}$ & $\begin{array}{l}\text { Molecular Cancer } \\
\text { Therapeutics }\end{array}$ & Torres et al. (2010) \\
\hline
\end{tabular}

Fonte: Elaboração própria com apoio do software HistCite a partir de dados da Web of Science.

As palavras-chave que foram citadas pelo menos 10 vezes foram selecionadas e estas correspondem à 15 dentre as 998 palavras-chave apresentadas, sob parâmetros do software VOSviewer. Para ilustrar graficamente a co-ocorrência das palavras mais citadas foi construída a Figura 2. 
Research, Society and Development, v. 10, n. 10, e96101018586, 2021

(CC BY 4.0) | ISSN 2525-3409 | DOI: http://dx.doi.org/10.33448/rsd-v10i10.18586

Figura 2: Co-ocorrência de palavras mais citadas e mais relacionados entre si (1998-2019).

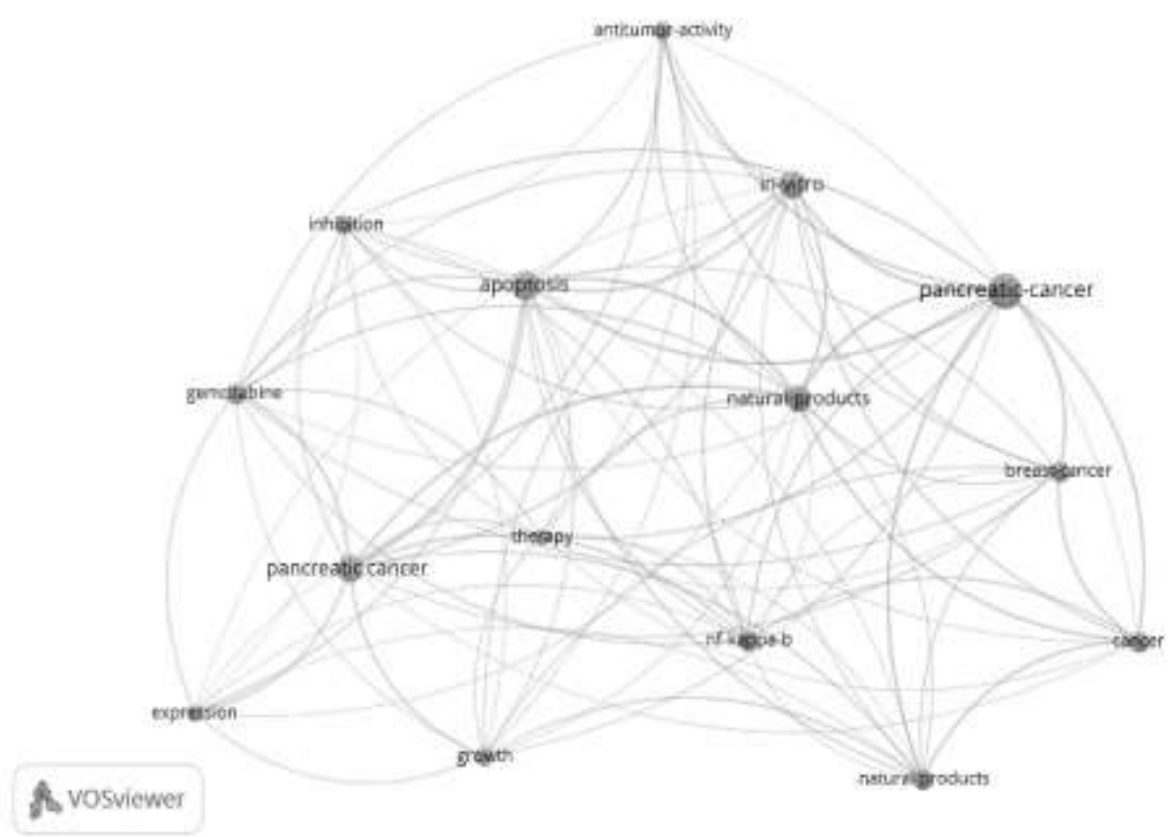

Fonte: Elaboração no software VOSviewer a partir de dados da Web of Science ${ }^{T M}$.

Análises bibliométricas representam um entendimento que fornece uma visão transversal e o estado atual dos trabalhos de pesquisa sobre o tópico de interesse. Trata-se de uma análise estatística e quantitativa que visa identificar o impacto acadêmico e as características das publicações em um campo específico de pesquisa, o que poderia fornecer informações úteis aos pesquisadores envolvidos no desenvolvimento de estratégias de pesquisa para abordar as questões de saúde.

Este estudo bibliométrico identificou documentos científicos em fontes acadêmicas que pesquisavam sobre o uso de produtos naturais como terapia para o câncer de pâncreas. A maioria dos documentos possui vários autores de diferentes instituições e nações colaboradoras, o que destaca a produtividade de atividades científicas. Além disso, as palavras-chave de pesquisa apresentadas nos dados bibliométricos refletem a complexidade e inclusão de várias disciplinas aliadas, como: biologia celular e molecular, farmacologia, oncologia, patologia, medicina clínica e saúde pública.

Embora haja grandes melhorias no tratamento de muitos tipos de câncer comuns na clínica (por exemplo, câncer de próstata e câncer de mama) nas últimas décadas, o câncer de pâncreas continua sendo o câncer mais mortal diagnosticado e representa um grande desafio (Mohammed et al., 2015). Este estudo pôde observar o crescente número de publicações sobre potenciais terapêuticas para esta patologia fazendo-se o uso de substâncias naturais.

Os remédios à base de plantas (fitoterápicos) têm sido usados para tratar várias doenças há milhares de anos em vários países, como China, Egito, Japão e Coréia, que passaram a ser aceitos como formas de medicamentos complementares e alternativos nos países ocidentais. Os produtos naturais, ou medicamentos fitoterápicos, desempenham papéis importantes na prevenção e terapia do câncer de pâncreas como uma abordagem adjunta promissora (Chen et al., 2008). Conforme apresentado pelos artigos mais citados (Tabela 4), eles foram creditados com vantagens substanciais, incluindo melhora ao sistema imunológico, aumento dos efeitos benéficos e diminuição dos efeitos colaterais da quimioterapia e radioterapia, supressão da progressão do tumor (Gaisina et al., 2009; Giannis, 2010; Gou et al., 2011; Heretsch, Tzagkaroulaki \& Giannis, 2010; Ko et al., 2017; Torres et al., 2010; Torres et al., 2012; Trembaly et al., 2009; Wang et al., 2009; Wyke, Russel \& Tisdale, 2004; Wang et al., 2009; Zang, 2005). 
Ao contrário da medicina ocidental, que geralmente usa compostos químicos purificados e tem como alvo parâmetros fisiológicos únicos, os produtos naturais geralmente consistem em múltiplos componentes e ervas que agem ou interagem simultaneamente por vários mecanismos de sinal celular e alvos moleculares (Chen et al., 2008).

Em 2010, Torres et al., sabendo que a glicoproteína mucina 4 de alto peso molecular (MUC4) é amplamente expressada no câncer de pâncreas e contribui para a regulação da diferenciação, proliferação, metástase e quimiorresistência de células cancerígenas pancreáticas, estudaram a timoquinona, extraída das sementes de Nigella sativa e mostrou excelentes propriedades antineoplásicas diminuindo a expressão da MUC4 e consequentemente sendo um potencial adjuvante no tratamento do cancer de pâncreas (Wang et al., 2009).

Ainda sobre proteínas amplamente expressadas no cancer de pâncreas, a Glycogen Synthase Kinase $3 \beta$ (GSK-3ß) também possui importante papel na proliferação tumoral nesta doença, e na busca por suprimir essa enzima, Gaisina et al desenharam e sintetizaram a partir de produtos naturais o benzofuran-3-yl-(indol-3-yl)maleimides que mostrou ser um potente inibidor da GSK-3 $\beta$ in vitro ${ }^{10}$. Recentemente, em 2019 um estudo clínico corroborou in vivo que os inibidores da GSK3b aumentam significativamente a morte tumoral quando combinados com a quimioterapia e radioterapia convencional (Ding et al., 2019).

Destinados a avaliar o potencial de mais um novo produto derivado de plantas como agente terapêutico para o câncer pancreático, Torres et al. em 2012 realizaram um extrato da graviola e verificaram a indução de necrose de células do câncer de pâncreas inibindo o metabolismo celular. A expressão de moléculas relacionadas à hipóxia e glicólise em células (HIF-1 $\alpha$, NF-кB, GLUT1, GLUT4, HKII e LDHA) foram regulados negativamente na presença do extrato. Esses parâmetros levaram a uma diminuição na tumorigenicidade e metástase de pacientes indicando características promissoras do produto natural contra essa doença (Torres et al., 2010).

O estudo publicado por Ko et al. em 2017 mostra que o Resveratrol presente nas uvas e no vinho mostrou efeitos citotóxicos in vitro contra diversos tipos de câncer, entre eles o câncer de pâncreas. A substância natural afeta uma variedade de estágios do câncer, da iniciação e promoção à progressão, afetando as diversas vias de transdução de sinal que controlam o crescimento e a divisão celular, inflamação, apoptose, metástase e angiogênese servindo como adjuvante ao tratamento do tumor no pâncreas (Ko et al., 2017).

Embora a análise dos dados desta revisão bibliométrica tenha sido bastante objetiva, a presença de limitações não pôde ser excluída. Primeiramente, a principal limitação refere-se ao idioma incluído no estudo, sendo apenas o inglês. Segundamente, o desenho do estudo dos clássicos de citação é limitado pelo tempo, desta forma, se um artigo de pesquisa de alta qualidade sobre o uso de produtos naturais como terapêutica para câncer de pâncreas foi publicado nos últimos anos, não podemos esperar que ele seja mais citado do que um artigo que existe há uma década ou mais. 
Tabela 4: Resumo dos 10 artigos citados do levantamento bibliográfico.

AUTOR (ANO) Wyke, Russel \& Tisdale

Zhang et al. (2005)

Wang et al. (2009)

Tremblay et al. (2009)

Gaisina et al. (2009)

Torres et al. (2010)

\section{Heretsch, Tzagkaroulaki \&} Giannis (2010)

TIPO DE ESTUDO

\section{Ensaio Clínico:}

"A indução da expressão do proteassoma no músculo esquelético é atenuada por inibidores da ativação de $N F-k B$ ",

Ensaio Clínico:

"O resveratrol inibe o acúmulo de fator $1 A$ indutivel por hipóxia induzido por hipóxia e a expressão de $V E G F$ em células de carcinoma espinocelular de língua humana $e$ hepatoma"

\section{Ensaio Clínico:}

"Novos ginsenósidos 25-OH-PPD e 25-OCH3-PPD como terapia experimental para câncer de pâncreas: atividade anticâncer $e$ mecanismos de ação"

Revisão Sistemática:

"Descoberta de um antagonista da via do ouriço potente e oralmente ativo (IPI-926)"

\section{Ensaio Clínico:}

"A síntese e a avaliação biológica de benzofuran-3-il (indol-3-il) maleimidas, potentes inibidores de GSK-3 que são superexpressados em carcinomas de cólon e pâncreas humanos, contribuindo para a proliferação e sobrevivência das células cancerígenas"

\section{Ensaio Clínico:}

"Efeitos da Timoquinona na Expressão da Mucina 4 nas Células do Câncer Pancreático: Implicações para o Desenvolvimento de Novas Terapias do Câncer"

\section{Revisão Sistemática:}

"Moduladores da via de sinalização hedgehog (hh) ",

\section{RESULTADOS}

Estes resultados sugerem que os agentes que inibem a translocação nuclear de inibidores da ativação do fator nuclear-kB podem ser úteis para o tratamento da perda de massa muscular na caquexia do cânce

Os resultados sugeriram que o fator $1 \mathrm{~A}$ indutível por hipóxia/ fator de crescimento endotelial vascular poderia ser um alvo promissor para o resveratrol no desenvolvimento de uma terapia quimiopreventiva e anticâncer eficaz em cânceres humanos

Os ginsenosídeos 25-OH-PPD e 25-OCH3-PPD podem representar novos agentes terapêuticos para o câncer de pâncreas. Ambos os compostos exerceram atividades anticâncer potentes in vitro e in vivo e levaram à regulação negativa de proteínas oncogênicas importantes e pró-crescimento / próproliferação.

Como resultado, a regressão completa do tumor foi observada em um Modelo de aloenxerto de meduloblastomab dependente de ouriço após administração oral diária

O tratamento de células de câncer pancreático com inibidores de glicogênio sintase quinase-3, 5 e 26, resultou na supressão da atividade de GSK-3 e uma diminuição distinta da expressão do inibidor de apoptose ligado ao X (XIAP), levando a apoptose significativa. Os presentes dados sugerem um possível papel dos inibidores de GSK-3 na terapia do câncer, além de suas aplicações mais proeminentes em distúrbios do SNC.

Os resultados indicam que o tratamento com timoquinona regulou negativamente a expressão de glicoproteína mucina 4 de alto peso molecular através da via proteassomal e induziu apoptose em células de câncer de pâncreas pela ativação de c-Jun NH2-terminal quinase e vias de proteína quinase ativada por mitógeno p38. Assim, a timoquinona tem potencial para o desenvolvimento de novas terapias contra o pâncreas

Câncer.

Estudos clínicos recentes mostraram que a sinalização de hh pode ser a base de uma nova classe importante de agentes terapêuticos com implicações de longo alcance em oncologia. Assim, a modulação da sinalização de hh por meio de pequenas moléculas surgiu como uma ferramenta 


\section{Ensaio Clínico:}

Gou et al.

(2011)

Ko et al.

(2017)

\section{Revisão sistemática:}

"Micelas poliméricas biodegradáveis carregadas com curcumina para terapia de câncer de cólon in vitro $e$ in vivo"

"O papel do Resveratrol na terapia do câncer" valiosa no combate a esses cânceres dependentes de hh.

As micelas de Cur / MPEG-PCL são uma excelente formulação aquosa injetável por via intravenosa de curcumina. Esta formulação pode inibir o crescimento do carcinoma do cólon através da inibição da angiogênese e matando diretamente as células cancerosas.

Um vasto corpo de estudos experimentais in vivo e in vitro e um poucos ensaios clínicos apresentaram evidências de que o resveratrol é capaz de atenuar os vários estágios da carcinogênese e possui grande potencial como um agente anticâncer, tanto para a prevenção quanto para a terapia de uma grande variedade de cânceres.

Fonte: Autores.

\section{Conclusão}

Pôde-se observar um crescimento significante no número de pesquisas sobre o uso de produtos naturais como terapia adjuvante ou neoadjuvante para o câncer de pâncreas e espera-se que, futuramente, muitas dessas terapias sejam utilizadas para ajudar os que necessitarem. A grande variedade de mecanismos de ação associados a compostos naturais é problemática em termos de isolamento e confirmação de alvos celulares específicos e seu impacto na sobrevivência de células tumorais. Embora estudos recentes tenham identificado alguns mecanismos de ação, estamos longe de entender todo o espectro de efeitos que a terapêutica natural exerce sobre as células normais e cancerígenas.

Desta forma evidencia-se a necessidade da crescente continuação de estudos acerca deste tema de importância para saúde pública, buscando aumentar a sobrevida e melhorar a qualidade de vida dos portadores de câncer pancreático

\section{Referências}

Atlas On-line de Mortalidade: Instituto Nacional de Câncer e Ministério da Saúde. https://www.inca.gov.br/MortalidadeWeb/pages/ Modelo01/consultar.xhtml.

Chen, S. T., Dou, J., Temple, R., Agarwal, R., Wu, K. M., \& Walker, S. (2008). New therapies from old medicines. Nature Biotechnology, $26(10)$, 1077-1083. http://dx.doi.org/10.1038/nbt1008-1077.

Ding, L., Madamsetty, V. S., Kiers, S., Alekhina, O., Ugolkov, A., Dube, J., Zhang, Y., Zhang, J.S., Wang, E., Dutta, S. K., Schmitt, D. M., Giles, F. J., Kozikowski, A.P., Mazar, A.P., Mukhopadhyay, D., \& Billadeau, D. D. (2019). Glycogen synthase kinase-3 inhibition sensitizes pancreatic cancer cells to chemotherapy by abrogating the TopBP1/ATR-mediated DNA damage response. Clinical Cancer Research, 25(21), 6452-6462. https://doi.org/ 10.1158/10780432.CCR-19-079.

Gaisina, I. N., Gallier, F., Ougolkov, A. V., Kim, K. H., Kurome, T., Guo, S., Holzle, D., Luchini, D. N., Bolnd, S. Y., Billadeu, D. D., \& Kozikowski, A. P. (2009). From a natural product lead to the identification of potent and selective benzofuran-3-yl-(indol-3-yl) maleimides as glycogen synthase kinase $3 \beta$ inhibitors that suppress proliferation and survival of pancreatic cancer cells. Journal of medicinal chemistry, 52(7), 1853-1863. http://dx.doi.org/10.1021/jm801317h.

Gou, M., Men, K., Shi, H., Xiang, M., Zhang, J., Song, J., Long J., Wang, Y., Luo, F., Zhao, X., \& Qian, Z. (2011). Curcumin-loaded biodegradable polymeric micelles for colon cancer therapy in vitro and in vivo. Nanoscale, 3(4), 1558-1567. http://dx.doi.org/10.1039/c0nr00758g.

Heretsch, P., Tzagkaroulaki, L., \& Giannis, A. (2010). Moduladores da via de sinalização do ouriço. Bioorganic \& medicinalochemical , 18 (18), 6613-6624. http://dx.doi.org/10.1016/j.bmc.2010.07.038.

Ko, J. H., Sethi, G., Um, J. Y., Shanmugam, M. K., Arfuso, F., Kumar, A. P., \& Ahn, K. S. (2017). The role of resveratrol in cancer therapy. International journal of molecular sciences, 18(12), 2589. http://dx.doi.org/10.3390/ijms18122589.

Luo, G., Zhang, Y., Guo, P., Ji, H., Xiao, Y., \& Li, K. (2019). Global patterns and trends in pancreatic cancer incidence: age, period, and birth cohort analysis. Pancreas, 48(2), 199-208. http://dx.doi.org/10.1097/mpa.0000000000001230.

Mohammed, A., Janakiram, N. B., Pant, S., \& Rao, C. V. (2015). Molecular targeted intervention for pancreatic cancer. Cancers, 7(3), 1499-1542. http://dx.doi.org/10.3390/cancers7030850. 
Research, Society and Development, v. 10, n. 10, e96101018586, 2021

(CC BY 4.0) | ISSN 2525-3409 | DOI: http://dx.doi.org/10.33448/rsd-v10i10.18586

Paulson, A. S., Cao, H. S. T., Tempero, M. A., \& Lowy, A. M. (2013). Therapeutic advances in pancreatic cancer. Gastroenterology, 144(6), 1316-1326. : http://dx.doi.org/10.1053/j.gastro.2013.01.078.

Perkhofer, L., Ettrich, T. J., \& Seufferlein, T. (2014). Pancreatic cancer: progress in systemic therapy. Gastrointestinal tumors, 1(4), 167-179. http://dx.doi.org/10.1159/000380785.

Siegel, R. L., Miller, K. D., \& Jemal, A. (2020). Cancer statistics, 2020. CA: a cancer journal for clinicians, 70(1), 7-30. https://doi.org/10.3322/caac.21590.

Souza, M. C.; Silva, M. D. \& Carvalho, R. (2010). Revisão integrativa: O que é e como fazer? Einstein, 8(1): 102-6.

Torres, M. P., Ponnusamy, M. P., Chakraborty, S., Smith, L. M., Das, S., Arafat, H. A., \& Batra, S. K. (2010). Effects of thymoquinone in the expression of mucin 4 in pancreatic cancer cells: implications for the development of novel cancer therapies. Molecular cancer therapeutics, 9(5), 1419-1431. http://dx.doi.org/10.1158/1535-7163.MCT-10-0075.

Torres, M. P., Rachagani, S., Purohit, V., Pandey, P., Joshi, S., Moore, E. D., \& Batra, S. K. (2012). Graviola: a novel promising natural-derived drug that inhibits tumorigenicity and metastasis of pancreatic cancer cells in vitro and in vivo through altering cell metabolism. Cancer letters, 323(1), 29-40. http://dx.doi.org/10.1016/j.canlet.2012.03.031.

Torres, M. P., Rachagani, S., Purohit, V., Pandey, P., Joshi, S., Moore, E. D., Johansson, S. L., Singh, P. K., Ganti, A. K., \& Batra, S. K. (2012). Graviola: A novel promising natural-derived drug that inhibits tumorigenicity and metastasis of pancreatic cancer cells in vitro and in vivo through altering cell metabolism. Cancer Letters, 323(1), 29-40. https://doi.org/10.1016/j.canlet.2012.03.031.

Tremblay, M. R., Lescarbeau, A., Grogan, M. J., Tan, E., Lin, G., Austad, B. C., Yu, L.-C., Behnke, M. L., Nair, S. J., Hagel, M., White, K., Conley, J., Manna, J. D., Alvarez-Diez, T. M., Hoyt, J., Woodward, C. N., Sydor, J. R., Pink, M., MacDougall, J., \& Campbell, M. J. (2009). Discovery of a Potent and Orally Active Hedgehog Pathway Antagonist (IPI-926). Journal of Medicinal Chemistry, 52(14), 4400-4418. https://doi.org/10.1021/jm900305z.

Wang, W., Rayburn, E. R., Zhao, Y., Wang, H., \& Zhang, R. (2009). Novel ginsenosides 25-OH-PPD and 25-OCH3-PPD as experimental therapy for pancreatic cancer: anticancer activity and mechanisms of action. Cancer letters, 278(2), 241-248. http://dx.doi.org/10.1016/j.canlet.2009.01.005.

Wyke, S. M., Russell, S. T., \& Tisdale, M. J. (2004). Induction of proteasome expression in skeletal muscle is attenuated by inhibitors of NF-к B activation. British journal of cancer, 91(9), 1742-1750. http://dx.doi.org/10.1038/sj.bjc.6602165.

Zhang, Q., Tang, X., Lu, Q. Y., Zhang, Z. F., Brown, J., \& Le, A. D. (2005). Resveratrol inhibits hypoxia-induced accumulation of hypoxia-inducible factor$1 \alpha$ and VEGF expression in human tongue squamous cell carcinoma and hepatoma cells. Molecular cancer therapeutics, 4(10), 1465-1474. http://dx.doi.org/10.1158/1535-7163.MCT-05-0198. 